\title{
Prototipo de sistema fotovoltaico conectado a red eléctrica: Diseño, caracterización e implementación
}

\section{Prototype of photovoltaic system connected to electrical network: Design, characterization and implementation}

\author{
MEJIAS-BRIZUELA, Nildia ${ }^{1} \dagger^{*}$, BRIÓN-GONZÁLEZ, Rosa ${ }^{2}$, RAMÍREZ-LUGO, Arturo ${ }^{3}$ y \\ OROZCO-GUILLÉN, Eber ${ }^{1}$ \\ Universidad Politécnica de Sinaloa, ${ }^{1}$ Unidad Académica de Ingeniería en Energía, ${ }^{2}$ Maestría en Enseñanza de la Ciencia, \\ ${ }^{3}$ Unidad Académica de Ingeniería en Mecatrónica
}

ID 1 ${ }^{\text {er }}$ Autor: Nildia, Mejias-Brizuela / ORC ID: 0000-0003-2973-473X, CVU CONACYT ID: 253092

ID 1 ${ }^{\mathrm{er}}$ Coautor: Rosa, Brión-González / ORC ID: 0000-0001-8116-5250, CVU CONACYT ID: 940784

ID $2^{\text {do }}$ Coautor: Arturo, Ramírez-Lugo / ORC ID: 0000-0002-5863-3105

ID $3^{\text {er }}$ Coautor: Eber, Orozco-Guillén / ORC ID: 0000-0001-9309-252X, CVU CONACYT ID: 252917

\section{Resumen}

Se presenta el diseño de un prototipo de un sistema solar fotovoltaico interconectado a red eléctrica basado en el Estándar del CONOCER EC 0586.01 con el cual estudiantes de Universidades Politécnicas y otras instituciones educativas con carreras de energías renovables y afines adquieran el conocimiento teóricopráctico de la tecnología debido a que es imperante ya este tipo de formación académica para lograr profesionales más competitivos. El diseño se realizó empleando el software SolidWorks ${ }^{\circledR}$, de dimensiones adaptables a pequeños espacios pequeños y de fácil transportación por su bajo peso. Su implementación consiste en las conexiones entre componentes y la correspondiente a red eléctrica y determinar la aceptación por los usuarios mediante cuestionarios aplicados. La caracterización radica en adquirir datos en tiempo real de parámetros físicos y eléctricos mediante herramienta computacional diseñada, las curvas características de paneles solares en función de la irradiancia solar recibida y gráficos de producción energética del sistema fotovoltaico. Finalmente el uso de la red eléctrica para abastecer una carga eléctrica no cubierta por el prototipo de sistema fotovoltaico interconectado.

Sistema fotovoltaico, Prototipo, Certificación

\begin{abstract}
We present the design of a prototype solar photovoltaic system interconnected to the electrical network based the Standard CONOCER Mexico EC 0586.01 with which students from Polytechnic Universities and other education institutes technological of renewable energy acquire the theoretical-practical knowledge of photovoltaic technology, for so that students professionals more competitive. The prototype is designed using SolidWorks ${ }^{\circledR}$ software, is design for small space by the dimensions that have and is easy transportation because it does not weigh. The implementation consist in connections between components and the corresponding connections to the electrical grid and determine the acceptance by users through questionnaires applied. The characterization consist in acquired real-time physical and electrics parameters by computational tool designed, the graphs of characteristic curves of solar panels as function of solar irradiance and graphics of energy production of the photovoltaic system. Finally the use of the electricity network to supply an electric demand not covered by the prototype of grid interconnected photovoltaic system.
\end{abstract}

Photovoltaic system, Prototype, Certification

Citación: MEJIAS-BRIZUELA, Nildia, BRIÓN-GONZÁLEZ, Rosa, RAMÍREZ-LUGO, Arturo y OROZCO-GUILLÉN, Eber. Prototipo de sistema fotovoltaico conectado a red eléctrica: Diseño, caracterización e implementación. Revista de Ingeniería Innovativa. 2019. 3-12: 1-8

*Correspondencia al Autor (Correo electrónico: nmejias@upsin.edu.mx)

$\dagger$ Investigador contribuyendo como primer Autor. 


\section{Introducción}

Dado que el territorio mexicano cuenta con una irradiación solar media diaria anual de 5.5 $\mathrm{kWh} / \mathrm{m}^{2} /$ día y superior a $8.0 \mathrm{kWh} / \mathrm{m}^{2}$ por formar parte del cinturón solar, desde el año 2014 es uno de los primeros cinco países más atractivos para la inversión extranjera en proyectos de energía solar fotovoltaica (ProMéxico, 2015) lo que ha permitido que el desarrollo de esta tecnología en los últimos años vaya en aumento.

De igual forma, el esquema de interconexión a la red eléctrica mediante balance neto para la generación de energía eléctrica fotovoltaica a pequeña escala (Secretaría de Energía, 2007) ha permitido por los beneficios que brinda un incremento en la generación distribuida, así en enero 2019 la capacidad de generación eléctrica fue de 496 MW basado en instalaciones en residencias y comercios, lo que implicó un incremento aproximado del $50 \%$ con lo instalado en 2018 (40 mil) las instalaciones realizadas en 2017 ( $27 \mathrm{mil})$ y mucho mayor respecto a las realizadas en el 2016 (12 mil) (Solar Depot, 2019). Al mismo tiempo, también se cuenta con una base sólida en la manufactura de paneles solares por empresas mexicanas y extranjeras (ProMéxico, 2015,2017).

Este crecimiento vertiginoso de la tecnología fotovoltaica motivó al gobierno mexicano y empresarios en el año 2015 a establecer un alto nivel de desempeño de los profesionistas y/o personas encargadas de las instalaciones para garantizar la competitividad económica, el desarrollo educativo y el progreso social, por lo que a través del Consejo Nacional de Normalización y Certificación de Competencias Laborales (CONOCER) establecieron el Estándar de Competencias EC 0586 y en el 2017 el EC 0586.01 que evalúa la metodología basada en normas y especificaciones técnicas mexicanas vigentes para la instalación de sistemas fotovoltaicos interconectados a red eléctrica en residencia, comercio e industria.

El fondo sectorial CONACyT-SENER brindó apoyo en el 2017-2018 para la certificación de estudiantes de energías renovables, profesores y técnicos de Universidades Politécnicas y Tecnológicas (ProMéxico, 2017).
La certificación en esta tecnología brinda a los estudiantes en energías uno de los beneficios más importante para todo joven que es una oportunidad sobresaliente de integrarse al mercado laboral y la movilidad en el mismo debido a que muchas empresas instaladoras con apoyo del Fideicomiso para el ahorro de energía eléctrica (FIDE) y transnacionales solicitan dicha certificación.

Bajo este contexto, las Universidades Politécnicas y Tecnológicas donde incursiona un gran número de jóvenes para estudiar ingeniería en energía o energías renovables deben garantizar el conocimiento práctico de estas tecnologías para el fortalecimiento del capital humano en función de las necesidades industriales, sociales y de crecimiento de país, pero no todas ellas cuentan con equipos e instrumentos para hacerlo dado que todavía son costosos aún más si son gabinetes integrados, por lo que es necesario desarrollar prototipos funcionales que garanticen tal fin.

El objetivo de este proyecto es el diseño y la implementación de un prototipo para la interconexión a red eléctrica de un sistema solar fotovoltaico (SFIR) que permita la enseñanza, formación y adquisición de competencias teórico-prácticas en estudiantes de energía referente a la generación de la energía fotovoltaica, su conversión y distribución y las formas de utilización de la tecnología.

A partir de la metodología de proyecto didáctico y la bottom-up se presenta el diseño del prototipo $\mathrm{y}$ de un pequeño programa computacional para la adquisición de datos y gráficos de parámetros importantes; la caracterización de componentes del SFIR, así como la generación de energía eléctrica y su aprovechamiento para la alimentación de cargas. Finalmente, la implementación del prototipo a través del acompañamiento pertinente del docente en el desarrollo de actividades prácticas por dos grupos de estudiantes del quinto cuatrimestre de ingeniería en energía. 
El prototipo está compuesto por módulos que se integran, tiene dimensiones y peso adaptable a pequeños espacios, componentes de calidad industrial, una estación de trabajo móvil con paneles solares a los que se adaptan equipos para medición de parámetros físicos y eléctricos, conectores de color para distinción de polaridad, interruptores, contador de energía, inversor para conexión a la red, cargas variables, medidor bidireccional, protecciones AC/DC, una caja de herramientas, manuales técnicos de los componentes, de instalación y de trabajo práctico que permiten desarrollar las competencias teórico-prácticas y laborales para cumplir los estándares de calidad.

Esta investigación pretende contribuir en el área de las energías renovables al desarrollo de prototipos didácticos de calidad como una estrategia de ahorro en comparativa con productos comerciales para la enseñanza de la tecnología solar fotovoltaica; como una estrategia atractiva para la incursión de estudiantes en investigación colaborativa y participativa; como una estrategia diferente de aprendizaje donde docente-alumno construyen juntos un nuevo conocimiento desde la particularidad de cada uno; como estrategia para la solución de problemas reales del área fotovoltaica; como herramienta para desarrollar la metodología de instalación descrita en el EC 0586.01 .

\section{Metodología}

Está basada en el "método de proyectos didácticos" con participación activa de estudiantes de ingeniería en mecatrónica e ingeniería en energía y profesores para llevar a cabo las etapas mencionadas, así como la evaluación en cada fase y en la totalidad del proyecto partiendo del saber hacer del estudiante pero también a partir de lo que necesita aprender.

\section{Diseño}

Es el eje central para llevar a cabo el prototipo seguido de la verificación del funcionamiento de sus componentes interconectados. Se basó en una metodología para diseños pequeños, ya que el prototipo de SFIR tiene una potencia nominal entre 500 y $1000 \mathrm{~W}$ y es la metodología BottomUp (Franco, M. 2017).
Previo, se realizó un prediseño que consistió en determinar las entradas (todos los componentes del SFIR, radiación solar y temperatura ambiente) y las salidas (cargas eléctricas como luminarias y/o electrodomésticos). El prototipo está conformado por tres partes que se integran mediante módulos.

1.- Una estación móvil compuesta por dos paneles solares fotovoltaicos silicio monocristalino de $250 \mathrm{~W} \pm 5 \%$ de potencia nominal fijado cada uno a una base metálica

2.- Tablero eléctrico que consiste en una lámina metálica sobre una base metálica fija, pintada con pintura electrostática y en la cual se integran los otros componentes fundamentales del SFIR: inversor de $600 \mathrm{~W}$ de potencia nominal para conexión a red eléctrica, medidor bidireccional de la Comisión Federal de Electricidad (CFE) Mexico, encargado de registrar la energía eléctrica producida por los paneles solares inyectada a la red de distribución de CFE así como la suministrada por la red para suplir cargas eléctricas que no cubre el SFIR (Galván G., 2019), cargas variables y su contador de energía, interruptores, conectores y protecciones eléctricas.

Para el diseño de este tablero se consideró las dimensiones y peso de cada componente integrante y se empleó el software SolidWorks ${ }^{\circledR}$ professional. Tiene dimensiones de aproximadamente $1.0 \mathrm{~m}$ de alto por $1.0 \mathrm{~m}$ de ancho lo que hace que pueda adaptarse a una mesa o en pequeños espacios de laboratorios de docencia y su peso hace que pueda transportarse fácilmente de un lugar a otro para efectos de certificación fuera de laboratorios. La Figura 1 muestra el diseño.

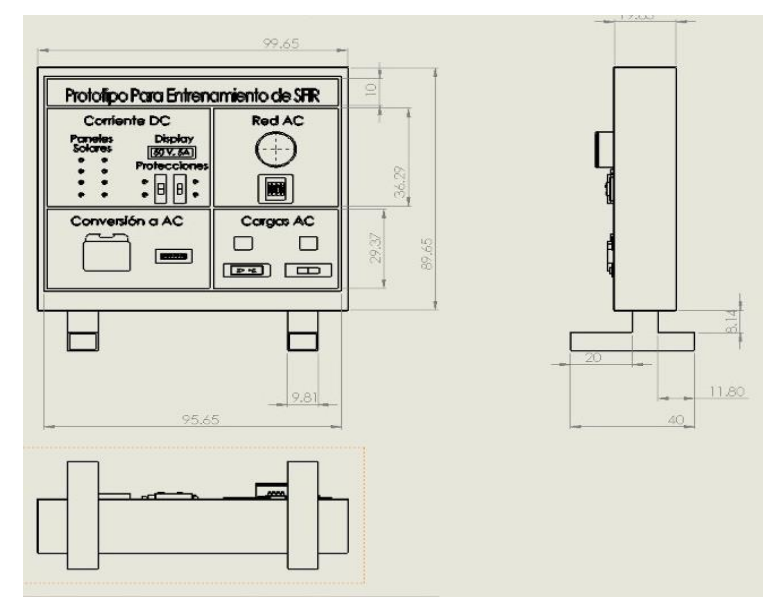

Figura 1 Diseño del tablero eléctrico que conforma el prototipo del SFIR

Fuente: Elaboración Propia

MEJIAS-BRIZUELA, Nildia, BRIÓN-GONZÁLEZ, Rosa, RAMÍREZLUGO, Arturo y OROZCO-GUILLÉN, Eber. Prototipo de sistema fotovoltaico conectado a red eléctrica: Diseño, caracterización e implementación. Revista de Ingeniería Innovativa. 2019. 
A su vez en la parte frontal se divide en cuatro módulos que se integran mediante cables de conexión: Corriente DC (corriente directa proveniente de los paneles solares), Conversión AC (Corriente alterna obtenida a través del inversor), cargas variables (luminarias, electrodomésticos) y red eléctrica (medidor bidireccional). Toda la demarcación se realizó mediante serigrafía.

Todos los componentes son de calidad industrial y dispuestos para conexión mediante las especificaciones establecidas en el estándar EC 0586.01 y en la NOM NOM-001-SEDE2018 de instalaciones eléctricas (CCNNIE y SENER, 2018).

La Figura 2 muestra el diseño de las conexiones eléctricas con sus respectivas protecciones del SFIR.

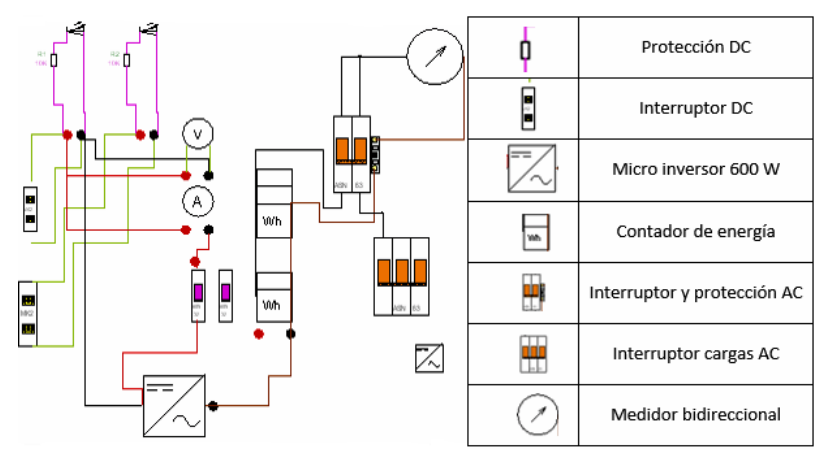

Figura 2 Diagrama eléctrico del prototipo de SFIR Fuente: Elaboración Propia

3.- Caja de herramientas con instrumentos de medición de mano tales como: brújula, inclinómetro, pinza amperimétrica DC/AC, multímetro y vatímetro digitales, desatornilladores y los manuales técnicos de los equipos (piranómetro, analizador de panel fotovoltaico, microinversor) de instalación del SFIR y el de actividades prácticas a realizar.

Para la adquisición y representación gráfica de datos experimentales arrojados por el prototipo se diseñó una herramienta computacional utilizando lenguaje de programación Python ejecutable en el entorno de programación Spyder (Lutz L., 2018). La Figura 3 muestra parte del código de programación.

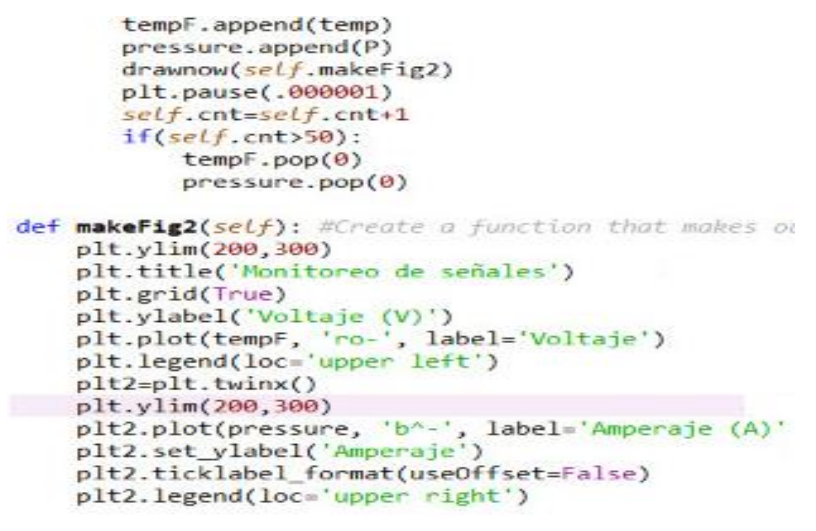

Figura 3 Código de programación utilizando Python para la adquisición de datos experimentales arrojados por el prototipo de SFIR

Fuente: Elaboración Propia

\section{Caracterización}

Se determinaron parámetros físicos y eléctricos de los paneles solares bajo condiciones de trabajo experimental, es decir, irradiancia solar y temperatura de celda diferentes a $1000 \mathrm{~W} / \mathrm{m}^{2} \mathrm{y}$ $25^{\circ} \mathrm{C}$ respectivamente medidos en días de verano a diferentes ángulos de inclinación. Para ello, se les fija un piranómetro portátil Kimo modelo SL 200 para medición de la irradiancia solar global captada una vez ubicados en un lugar libre de sombras y orientados al sur.

También se les conecta un analizador fotovoltaico (Prova 210) para determinar parámetros eléctricos básicos (Vmaxp, Imaxp, $\mathrm{Vca}$, Icc) y la curva característica corrientevoltaje (IV), potencia-voltaje (PV). Mediante la herramienta computacional se obtuvieron datos ambientales de los paneles solares (temperaturapresión) y la puesta en marcha del SFIR determinando la energía consumida por las cargas variables anexadas a través del contador de energía y el funcionamiento del mismo a través del medidor bidireccional.

\section{Implementación}

El prototipo se dispuso en una mesa del laboratorio de energía y la implementación se realizó a través de una prueba piloto que involucro dos grupos de estudiantes (60 en total) de la asignatura Sistemas Fotovoltaicos con Laboratorio del quinto cuatrimestre de Ingeniería en Energía. El grupo 1, previo a realizar trabajo práctico de instalación conforme al estándar EC 0586.01 y mediciones eléctricas en un SFIR de $10 \mathrm{~kW}$ de potencia nominal ubicado en techo de edificio de la Universidad, tuvo entrenamiento en el tablero haciendo uso de los manuales correspondientes.

MEJIAS-BRIZUELA, Nildia, BRIÓN-GONZÁLEZ, Rosa, RAMÍREZLUGO, Arturo y OROZCO-GUILLÉN, Eber. Prototipo de sistema fotovoltaico conectado a red eléctrica: Diseño, caracterización e implementación. Revista de Ingeniería Innovativa. 2019. 
Mientras que, el grupo 2, fue directamente a actividades prácticas en el SFIR de $10 \mathrm{~kW}$ de potencia nominal y luego al tablero de entrenamiento del prototipo. Para la evaluación se emplearon como técnicas y/o instrumentos de investigación la entrevista, la observación y el cuestionario.

La entrevista se realizó a los profesores de la asignatura teórica y la asignatura práctica relacionada con los sistemas fotovoltaicos y asignaturas con contenido de circuitos eléctricos para determinar lo relevante a evaluar en las rúbricas y cuestionarios aplicados a los usuarios del prototipo. La observación, cuyos instrumentos de investigación fueron registros escritos por parte de los estudiantes, fotografías, videos y rúbricas de evaluación a los usuarios y el cuestionario para determinar la escalas de opinión de los usuarios del prototipo de SFIR.

Primero se aplicó a todos los estudiantes de los dos grupos de trabajo una rúbrica que evalúo por parte de un grupo de profesores certificados en el EC 0586.01 el desempeño de los estudiantes en función de los saberes aprendidos y después se aplicó un cuestionario diseñado en función de la escala de Likert (Méndez L., Peña J., 2007), con 10 preguntas a un grupo de 32 estudiantes seleccionados al azar del total inscrito en la asignatura y con una duración de 10 minutos. Los datos colectados fueron tabulados y tratados para realizar análisis estadístico descriptivo.

\section{Resultados}

La Figura 4 muestra el diseño del prototipo del sistema fotovoltaico interconectado a red eléctrica con todos los componentes que los integran y los módulos de trabajo.

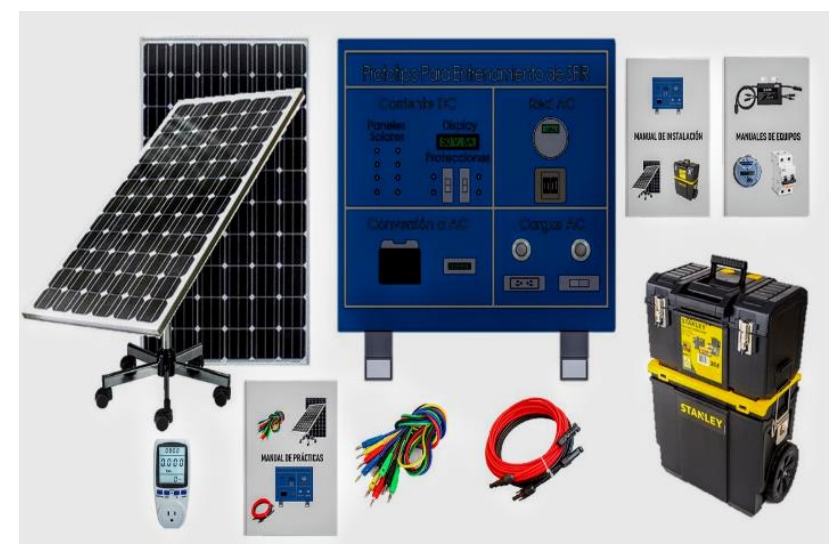

Figura 4 Prototipo de sistema fotovoltaico interconectado a red eléctrica con todos los componentes que lo integran Fuente: Elaboración Propia
La Figura 5 presenta la interfaz gráfica que permite almacenar parámetros ambientales y eléctricos en tiempo real y su respectiva gráfica o exportarlos a un archivo Excel para que el estudiante construya el gráfico.

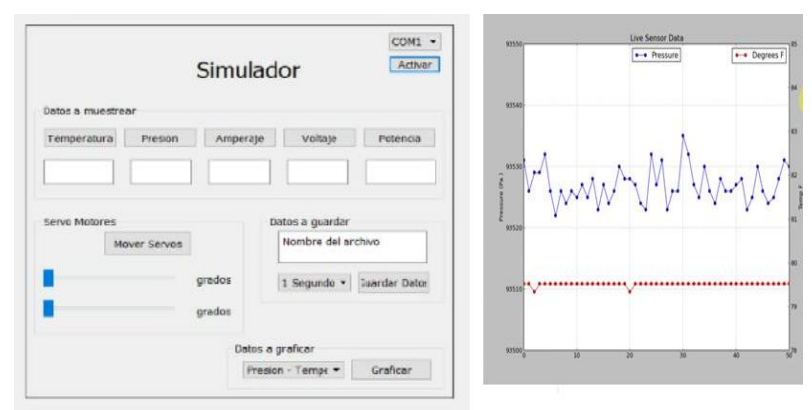

Figura 5 Interfaz gráfica para adquisición de parámetros Fuente: Elaboración Propia

La caracterización de la estación móvil del prototipo se muestra en el Gráfico 1, Figura 6 y Figura 7. El Gráfico 1 determina la relación que existe entre la energía eléctrica fotovoltaica generada y la irradiancia solar captada por uno de los dos paneles solares base silicio monocristalino durante un tiempo determinado en un día de verano (soleado-nublado) para posteriormente ser entregada a la red eléctrica.

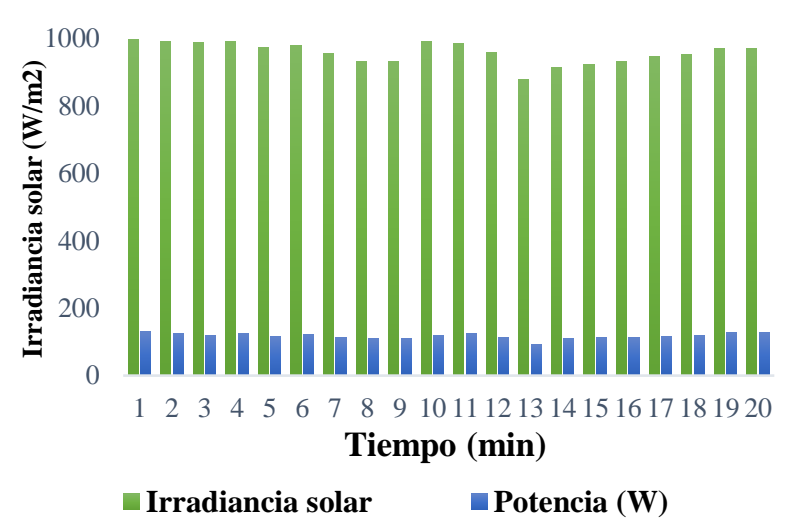

Gráfico 1 Potencia producida por un panel solar del prototipo de SFIR monocristalino de $250 \mathrm{~W}$ respecto a la irradiancia solar captada

Fuente: Elaboración Propia

Ambas mediciones se determinaron con el piranómetro portátil y el Prova 210 respectivamente a una temperatura promedio ambiental de $30^{\circ} \mathrm{C}$ :

La Figura 6 muestra la curva IV, mientras que, la Figura 7 muestra la curva PV del panel solar base silicio monocristalino seleccionado, medidas ambas a diferentes valores de irradiancia solar y temperatura promedio ambiental de $30^{\circ} \mathrm{C}$ y ángulo de inclinación fijo. 
Se muestra que tanto la corriente como la potencia eléctrica son directamente proporcional a la radiación solar incidente sobre el panel solar, mientras que el voltaje es aproximadamente constante. Esto le permite al estudiante corroborar los conocimientos teóricos impartidos en el aula de clases.



Figura 6 Curva IV característica de panel solar monocristalino de $250 \mathrm{~W}$ perteneciente a la estación móvil del prototipo de SFIR respecto a la irradiancia solar captada a $30^{\circ} \mathrm{C}$ y ángulo de inclinación fijo Fuente: Elaboración Propia

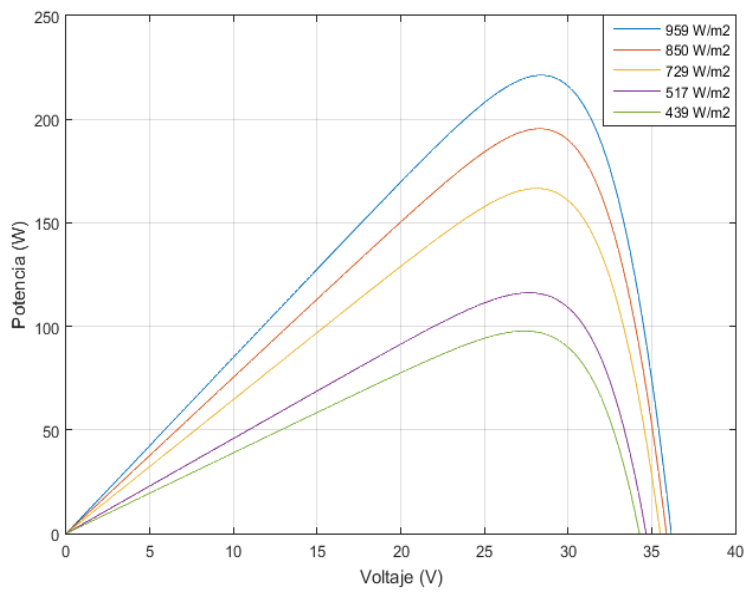

Figura 7 Curva PV característica de panel solar monocristalino de $250 \mathrm{~W}$ perteneciente a la estación móvil del prototipo de SFIR respecto a la irradiancia solar captada a $30^{\circ} \mathrm{C}$ y ángulo de inclinación fijo Fuente: Elaboración Propia

La caracterización referente a la puesta en marcha de todos los componentes que integran el prototipo de SFIR se realizó conectando cargas variables, una DC compuesta por dos lámparas de $75 \mathrm{~W}$ cuyo consumo eléctrico pueda ser suministrado por la energía eléctrica generada por el SFIR y la otra.
Un artefacto eléctrico (cafetera con una potencia de $800 \mathrm{~W}$ ) cuyo consumo eléctrico sea ahora suministrado por la red eléctrica de CFE con el fin de que el estudiante adquiera el conocimiento de las aplicaciones de la tecnología fotovoltaica, excedentes suministrados a red, ventajas, desventajas, la interconexión a red eléctrica, todo a partir de las capacidades desarrolladas durante las experiencias prácticas realizadas.

El Gráfico 2 muestra la energía eléctrica generada por los dos paneles solares de la estación móvil del prototipo de SFIR, el consumo eléctrico del artefacto y la energía suministrada por la red eléctrica para compensar la potencia real del artefacto.

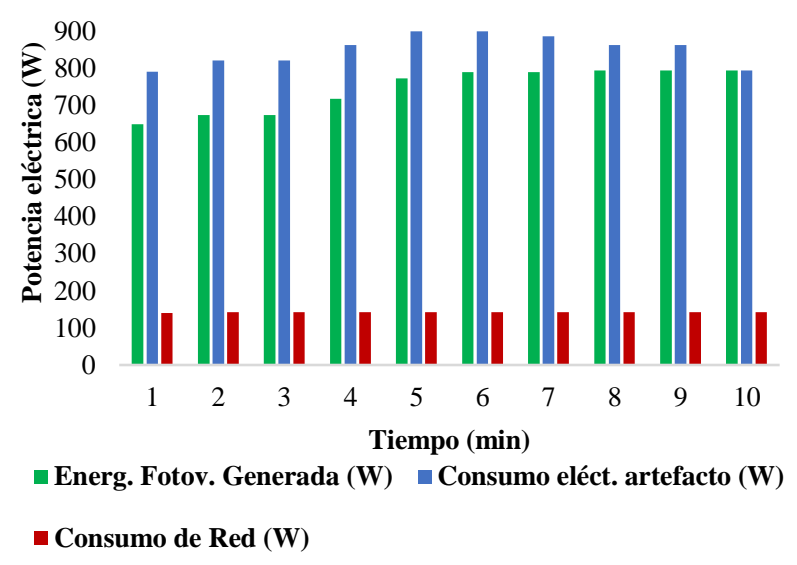

Grafico 2 Caracterización del prototipo de SFIR a través del consumo eléctrico de un artefacto cubierto tanto por los paneles solares del y la red eléctrica CFE

Fuente: Elaboración Propia

Referente al impacto en los dos grupos de trabajo al utilizar el prototipo a partir de las actividades prácticas realizadas se tiene el siguiente análisis descriptivo de acuerdo al tratamiento estadístico de los datos

1.- El $90 \%$ de los estudiantes encuestados manifiestan estar completamente de acuerdo con el entrenamiento realizado en el prototipo porque adquirió las capacidades y habilidades prácticas requeridas de manera sencilla por el conocimiento previo de los circuitos eléctricos, conexión de componentes, etc. descritos en el manual de prácticas y de instalación, lo que le brindo confianza y motivó para llevar a cabo la instalación fotovoltaica interconectada a red de $10 \mathrm{~kW}$ de potencia nominal mencionada $\mathrm{y}$ cualquier otra instalación, además de emplear un tiempo de instalación corto. 
Por lo tanto, generaron un mayor interés en emplearse en aplicaciones y desarrollo de la tecnología fotovoltaica y en la certificación del EC 0586.01. Al mismo tiempo el profesor de la asignatura se le facilitó la evaluación de conocimientos prácticos y manifestó que los estudiantes que primero se entrenaron con el prototipo no presentaron ningún problema para desarrollarla.

2.- El $10 \%$ restante, manifestó estar de acuerdo con el entrenamiento previo en el prototipo pero que se les dificultó un poco la instalación por ser un tablero pequeño ya que preferían el sistema fotovoltaico de $10 \mathrm{~kW}$ sin importarles el tiempo que se tomaran para hacerlo y su exposición al sol. Sin embargo, se les hizo interesante porque se asocian cargas eléctricas variables que permiten observar las ventajas de un SFIR, hecho que la instalación de $10 \mathrm{~kW}$ no tiene. De igual manera manifestaron no tener interés a corto plazo de la certificación.

\section{Agradecimiento}

Al PRODEP por el financiamiento otorgado al Cuerpo Académico de Ingeniería en Energía para el desarrollo del proyecto "Desarrollo y evaluación de prototipos de sistemas de generación de energía no convencional (eólica, solar y biomasa) para la formación de recurso humano".

\section{Conclusiones}

El prototipo presentado es un modelo diseñado fundamentalmente para facilitar las actividades prácticas relacionadas con la tecnología solar fotovoltaica mediante la obtención y representación gráfica de los parámetros esenciales de la misma, cálculos matemáticos para la obtención de energía eléctrica en un determinado tiempo, el ahorro económico que representa y el tiempo de amortización. Todo ello hace que los usuarios sin complicación se acoplen a la normatividad mexicana, por lo que también está diseñado para cuantificar el nivel de aceptación entre cada uno de ellos.

Brinda a estudiantes, profesionales afines a la energía y personas no profesionales interesadas en adquirir conocimientos en la tecnología fotovoltaica de interconexión a red eléctrica una oportunidad de realizar una instalación solar real donde se pueden cometer errores y buscar una solución.
Lo que representa una parte fundamental para el desarrollo de capacidades educativas, confianza y motivación para el trabajo eléctrico, para el trabajo colaborativo en equipo, etc.

La participación de herramientas computacionales para el diseño y la adquisición de datos en tiempo real también es fundamental en el desarrollo de prototipos tecnológicos debido a las modificaciones que estos experimentaran a largo plazo.

Los trabajos a futuro conllevan a hacer trasferencia a Universidades Tecnológicas, Institutos Tecnológicos y Preparatorias presentes en la localidad con carreras de Energías para que hagan uso del prototipo y las observaciones correspondientes que a bien tengan para la mejora continua del mismo.

Aun cuando ya fue usado por un grupo de 14 personas (no estudiantes) para certificación del EC 0586.01, es conveniente seguir utilizándolo para tomar en cuenta las observaciones y mejoras que puedan aportar los participantes en este evento. De igual manera se pretende diseñar la parte de sistemas fotovoltaicos autónomos que se integraría al prototipo.

\section{Referencias}

Comité Consultivo Nacional de Normalización de Instalaciones Eléctricas y Secretaría de Energía. Proyecto de Norma Oficial Mexicana PROY-NOM-001-SEDE-2018, Instalaciones Eléctricas (utilización). Diario Oficial de la Federación (2018, 06 de agosto). Mexico. Recuperado de http://dof.gob.mx.

Consejo Nacional de Normalización y Certificación de Competencias Laborales (2017). Instalación de sistemas fotovoltaicos en residencia, comercio e industria. Recuperado de https://www.conocer.gob.mx.

Franco Avendaño, M. A. (2017). Diseño $e$ implementación de un sistema alterno de suministro eléctrico mediante energía solar fotovoltaica en iluminación y térmica en calentamiento de agua en una casa rural en el municipio de Icononzo Tolima (Tesis de Licenciatura). Universidad Pedagógica Nacional, Bogotá Colombia. 
Fideicomiso para el ahorro de energía eléctrica. Certifícate con FIDE en el Estándar EC 0586.01 Instalación de sistemas fotovoltaicos en residencia, comercio e industria. Recuperado de http://www.fide.org.mx.

Galván G. (2019). Funcionamiento del medidor bidireccional de CFE. Recuperado de https://cceea.mx/

Lutz L. (2018). Python Guía paso a paso para aprender programación Python. España: Python.

Méndez Hinojosa L. M., Peña Moreno J. A. (2007). Manual práctico para el diseño de la Escala de Likert. México: Trillas.

ProMéxico (2015). Energías renovables (Publicación $\mathrm{N}^{\circ}$ 2). México, D.F. Recuperado de http://www.promexico.gob.mx.

ProMéxico (2017). Energías renovables y electricidad (Publicación $\mathrm{N}^{\circ}$ 1). México, D.F. Recuperado de http://www.promexico.gob.mx.

Secretaría de Energía (2007). Resolución $\mathrm{N}^{\circ}$ RES/176/2007. Diario Oficial de la Federación. (2007, 27 de junio). Mexico. Recuperado de http://dof.gob.mx.

Solar Depot México (2018, 19 de diciembre). Más de 40 mil instalaciones fotovoltaicas en México en 2018. Recuperado de http://www.sde.mx. 